\title{
Apertureless near-field optical microscopy: Differences between heterodyne interferometric and non-interferometric images
}

\author{
Ruben ESTEBAN, ${ }^{1,2,3}$ Ralf VOGELGESANG, ${ }^{1}$ and Klaus $\mathrm{KERN}^{1,4}$ \\ ${ }^{1}$ Max Planck Institut für Festkörperforschung, 70569 Stuttgart, Germany \\ ${ }^{2}$ Centro de Física de Materiales, Centro Mixto CSIC-UPV/EHU \\ and Donostia International Physics Center (DIPC), \\ 20018 Donostia-San Sebastián, Spain \\ ${ }^{3}$ Laboratoire Charles Fabry, Institut d'Optique, \\ CNRS, Université Paris-Sud, Campus Polytechnique, \\ RD 128, 91127 Palaiseau Cedex, France \\ ${ }^{4}$ Institut de Physique des Nanostructures, \\ Ecole Polytechnique Fédérale de Lausanne, CH-1015 Lausanne, Switzerland
}

\begin{abstract}
We analyze simulations of the image generation process in apertureless scanning near-field optical microscopy to study the differences between heterodyne interferometric and non-interferometric detection schemes implemented in experiments. Beyond the well-known possibilities of interferometry to enhance signals and extract optical phase information, we find that heterodyne interferometric detection may in fact lead to more robust and easier to interpret experimental results. Further, our results illustrate how simulations that do not include the use of interferometric detection in a a given experiment can lead to significant disagreements with the measurements.
\end{abstract}

Keyworkds: Apertureless near field optical microscopy, interferometry, demodulation, simulations, imaging 


\section{INTRODUCTION}

The possibly best known limitation to the wealth of information achievable with traditional optical microscopy is the classical diffraction limit. However, different techniques under current development can overcome this limitation. We focus in this paper on apertureless Scanning Near Field Optical Microscopy $(a S N O M)[1,2]$.

$a S N O M$ typically uses a sharp, oscillating tip to gain information about the sample with lateral resolution in the order of the tip apex radius. In an active configuration[3, 4], the tip apex interacts strongly with a closely situated substrate and gives rise to intense and very localized near fields. The near fields are, however, not directly accessible, and image construction relies on the detection of scattered far fields. A first crucial aspect is thus the relationship between the near field interaction and the scattered fields[5]. The method used to detect and analyze such far fields is also relevant. For example, demodulation at the higher harmonics of an oscillating tip has been shown useful to improve lateral resolution and suppress background[6]. The background, for example from the bulk or the tip, complicates the discriminatation of the high resolution information and its suppression is convenient for experimental interpretation.

In recent studies the elastically scattered radiation was detected using both interferometric $[7,8]$ and non interferometric [9-11] schemes. These two schemes are not equivalent, though, and the differences go beyond the possibility to obtain a stronger signal and phase information with the former. The differences have been qualitatively discussed and experimentally shown $[12,13]$. To better understand the differences, we start by considering the recorded signal $S_{n}$ obtained from demodulation with a dual-phase lock-in amplifier at the $n$-th harmonic of the oscillation angular frequency $\omega$

$$
S_{n} \propto \frac{1}{T} \int_{0}^{T} I_{d e t} e^{i\left(n \omega t+\Theta_{l i}\right)} d t,
$$

$T=2 \pi / \omega$ is the oscillation period and $\Theta_{l i}$, zero in the following if not mentioned otherwise, allows to introduce the internal phase (relative to the tip oscillation) of the lock-in amplifier. $I_{\text {det }}$ is the current generated at the photodetector, and it differs between interferometric and non-interferometric schemes[14]. For the latter case and under convenient conditions it takes the form

$$
I_{d e t} \propto \int_{A_{d e t}}\left|E_{s c a}\right|^{2} d A_{d e t}
$$


$A_{d e t}$ is the area of the detector, oriented perpendicular to the propagation direction of the detected radiation. The complex-valued $E_{s c a}=\left|E_{\text {sca }}\right| \exp \left(i \Theta_{\text {sca }}\right)$ refers to the electrical field of the signal scattered by the tip-substrate system. To illustrate one significant difficulty that appears in non-interferometric detection, we consider a sample consisting of an object with deep-subwavelength structure supported by a substrate, which itself hardly varies spatially and only on a scale of a wavelength or more.

Intuitively, it is appealing to introduce a conceptual decomposition of the scattered fields[13]. With $E_{o b j}=\left|E_{o b j}\right| \exp \left(i \Theta_{o b j}\right)$ we refer to components that stem from the interaction between the tip apex and the sub-wavelength objects. The remainder, $E_{b k g}=$ $\left|E_{b k g}\right| \exp \left(i \Theta_{b k g}\right)$, may be considered a slowly varying "background". $E_{o b j}$ and $E_{b k g}$ are related to those spatial frequencies in the recorded images that are higher and lower than roughly half a wavelength. Only $E_{o b j}$ carries high spatial resolution information. But it is difficult, if not impossible, to give an exact, fully satisfactory algebraic definition and we use the terms $E_{o b j}$ and $E_{b k g}$ here merely to facilitate the discussion, without attempting a rigorous distinction. Nevertheless, one important conclusion can be drawn immediately even from this conceptual distinction. A self-homodyne term $2\left|E_{b k g}\right|\left|E_{o b j}\right| \cos \left(\Theta_{b k g}-\Theta_{o b j}\right)$ occurs in the total scattered field, $\left|E_{s c a}\right|^{2}=\left|E_{b k g}+E_{o b j}\right|^{2}$, which mixes both contributions. The strength and phase of $E_{b k g}$ thus affect the value of Eq. (1) in a non-trivial way for non-interferometric detection. In general, this will complicate image interpretation even if $E_{b k g}$ would be perfectly constant [15].

We now consider an interferometric detection scheme. That is, before impinging onto the photodetector, the scattered signal interferes with a collinear, planar wave $E_{r e f}^{p}=$ $\left|E_{r e f}^{p}\right| e^{i \Theta_{r e f}^{p}}$. Considering $E_{r e f}^{p}$ constant and sufficiently large, the relevant $I_{\text {det }}$ expression for harmonic $n>0$ is

$$
I_{d e t} \propto \int_{A_{d e t}}\left|E_{r e f}^{p}\right|\left|E_{s c a}^{p}\right| e^{i\left(\Theta_{r e f}^{p}-\Theta_{s c a}^{p}\right)} d A_{d e t}
$$

Only the interferometric term appears because the constant contribution $\left|E_{r e f}^{p}\right|^{2}$ will be filtered out by the harmonic demodulation and we assume the term $\left|E_{s c a}^{p}\right|^{2}$ negligibly small. The superindex $p$ indicates the polarization selected with the reference beam. The complex exponential phase factor in the integrand corresponds to homodyne in quadrature, heterodyne, balanced homodyning, phase-shifting or related interferometric techniques $[14,16,17]$. The expressions already indicate that interferometry gives access to optical phase informa- 
tion, and that the presence of $\left|E_{r e f}^{p}\right|$ allows to boost the detected signal.

A superficial look at the integrands of Eqs. $(2,3)$ might suggest an otherwise simple relation between the module of the non-interferometric and heterodyne interferometric signals, with the differences related to the presence of $\left|E_{s c a}^{p}\right|^{2}$ or $\left|E_{s c a}^{p}\right|$, respectively. However, the presence of the phase $\Theta_{o b j}^{p}$ renders the differences more intrincate. For example, the phase modulation due to the tip oscillation affects also the demodulated signal amplitude $\left|S_{n}\right|$ $[6,14,18,19]$. If we explicitly decompose $E_{s c a}^{p}=E_{o b j}^{p}+E_{b k g}^{p}$ for the interferometric case as we did for non-interferometric detection, the relevant integrand in Eq. (3) turns into

$$
\left(\left|E_{o b j}^{p}\right| e^{-i \Theta_{o b j}^{p}}+\left|E_{b k g}^{p}\right| e^{-i \Theta_{b k g}^{p}}\right)\left|E_{r e f}^{p}\right| e^{i \Theta_{r e f}^{p}}
$$

in this case, the simple additive form seems promising for filtering out the slowly varying $E_{b k g}^{p}$, for example by the high harmonic demodulation in Eq. (1), and thus better discriminating the high resolution information contained in $E_{o b j}^{p}$. Notice that taking the module $\left|S_{n}\right|$ of the recorded signal does reintroduce a certain cross-talk between both contributions. Thus, it may be better to consider the full complex signal, for example, if the recorded image is spatially filtered in a post-processing step.

These considerations and previous work $[12,20]$ suggest that the different dependence of $\left|S_{n}\right|$ on tip position results in clearer images for heterodyne interferometric than for noninterferometric measurements. To our knowledge, a detailed quantitative account, however, has yet to be given. Numerical simulation[10, 21] of scenarios closely following the experimental conditions is a challenging prerequisite. In particular, it appears necessary to model large probe tips[22, 23] which may be interacting strongly with the sample. It is also not sufficient to compute only the near fields or the total scattering cross section. The amplitude and phase distribution of the electric fields over the detector area must be computed, and the simulations must capture accurately the behavior of at least the first few derivatives of the signal $I_{d e t}$, as they play a key role in the value of the different harmonics $S_{n}$.[6, 14]

In our recent work, we have developed simulations that consider these aspects[22], but we did not analyze the influence of interferometry on the resulting quantities. In the following we simulate approach curves and one-dimensional scans and infer different qualitative features of non-interferometric and heterodyne interferometric detection schemes relevant to experimental and theoretical work. 


\section{GEOMETRY AND SIMULATIONS}

For our simulations we use a multiple multipole method, as implemented in the numerical platform MaX-1[24]. It employs expansions that are vectorial solutions (or excellent numerical approximations) of the Maxwell equations. MaX-1 minimizes the error at the boundaries, in our case to less than 0.5 percent average value. Moderately increasing the number of free parameters used to minimize the error did not significantly alter the results of this paper, which supports a good numerical convergence of the obtained results.

A silicon tip illuminated by an external excitation oscillates along the vertical $z$ direction parallel to its axis, with $z$ decreasing as the tip approaches the top of a sample. Fig. 1(a) sketches the geometry with one sample consisting of a glass sphere of $2 \mu m$ diameter containing fully a $10 \mathrm{~nm}$ radius gold spherical inclusion, the center of both aligned along $z$ and their upper point at $1 \mathrm{~nm}$ distance. Fig. 1(b) represents the fields for this geometry and a particular tip position and will be, together with Fig. 1(c), explained more in detail later on. We will be also interested in a large homogeneous gold sphere of the same $2 \mu \mathrm{m}$ diameter and in two smaller gold spheres in vacuum (Fig. 4(a)), of 10 and 30nm radius, their top surface at the same $z$ position and their centers separated $60 \mathrm{~nm}$ in the $x$ direction; the $x$ direction is perpendicular to $z$ and tangent to the different spheres at their upper point, with $x z$ containing the tip axis. The origin of the coordinate axis is at the upper point of the gold substrate or $1 \mathrm{~nm}$ over the $10 \mathrm{~nm}$ gold inclusions. To minimize the effect in non-interferometric detection of the self-homodyne term $\left|E_{b k g}\right|\left|E_{o b j}\right| \cos \left(\Theta_{b k g}-\Theta_{o b j}\right)$ from a constant contribution from an extended substrate, we subtract the fields scattered by the bare $2 \mu \mathrm{m}$ large spheres, either glass or gold, when illuminated in absence of any probing tip or inclusion.

The modeled tip is a $C^{(1)}$ continuous cone with cylindrical symmetry, capped by two hemispheres. The smaller hemisphere probes the sample and has a radius $R=10 \mathrm{~nm}$. $1400 \mathrm{~nm}$ long tips are used for the homogeneous gold sample, while the tip is 500 $\mathrm{nm}$ long for the other substrates. The cone half angle, i.e the angle between the generatrix and the axis, is $10^{\circ}$. The excitation makes a 70 degree angle with the axis of the tip, it is p (TM) polarized with $x z$ being the plane of incidence and its wavelength $\lambda$ is $\sim 514.5 \mathrm{~nm}$. At this frequency, $\epsilon=2.1,17.76+0.508 i$ and $-3.95+2.58 i$ for the glass, silicon[25] and gold[26] respectively. Simple planar waves excite the $500 \mathrm{~nm}$ long tips, but we use a 5 th order corrected gaussian 

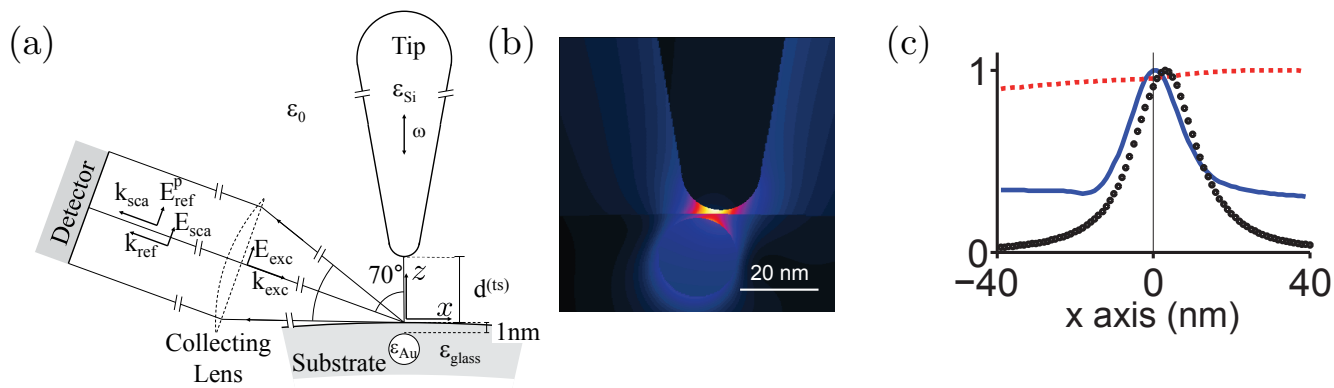

FIG. 1: (a) One of the considered geommetries, illuminated by an external p-polarized plane wave with electric field $E_{\text {exc }}$. Here, the substrate is a $1 \mu \mathrm{m}$ glass sphere and, $1 \mathrm{~nm}$ below its upper point, there is a $10 \mathrm{~nm}$ radius gold spherical inclusion. The orthogonal coordinate system is placed directly at the top of the large sphere, with the $x$ axis tangential to the sphere at this point, and $z$ parallel to the tip axis. The axis is contained in $x z$, the plane of incidence of the illumination, and the scanning direction is $x$. The upper point of the gold inclusion and large glass sphere corresponds thus to $x=0, z=-1 n m$ and $x=z=0$ respectively. The lens is considered for the collection of the fields, and for heterodyne interferometric detection the scattered signal $E_{\text {sca }}$ is mixed with a reference beam $E_{r e f}^{p}$ that selects the p polarized ( $x z$ plane) component $E_{s c a}^{p}$. (b) Example of the electrical field modulus in the proximity of the apex for the geommetry in (a). The strongest fields, in white, are $\sim 27.6$ times larger than the excitation field. The color scale is linear with the black corresponding to zero field strength. (c) Various signal scan tracks for the same geommetry. The black circles correspond to the near fields, in the absence of a tip, at $1 \mathrm{~nm}$ of the substrate as a function of $x$ position. The solid blue line refers to the near field enhancement when a nonoscillating tip scans the sample along $x$ at a constant $1 \mathrm{~nm}$ distance. The red dashed line shows the signal $I_{\text {det }}$ detected non-interferometrically under the same conditions. For clarity, all traces are normalized to the maximum value displayed. (b) and (c) do not include the fields from the excitation or scattered by the homogeneous glass substrate standing in isolation

beam [27] for the $1400 \mathrm{~nm}$ long tips to avoid strong illumination of the larger hemisphere. The waist radius of the gaussian beam is $500 \mathrm{~nm}$ and its focus center is at the top of the gold substrate.

The signal is detected by a dual-phase lock-in amplifier at a higher harmonic of the frequency of oscillation $\omega$ of the probing tip, according to Eq. (1). During the oscillation 
the $z$ position of the lower apex point follows $z^{(t)}=z_{\text {min }}+A(1+\cos (\omega t))$, where $A$ is the oscillation amplitude. The value of $\omega$ does not influence the final result. $z_{\min }$ is the corresponding $z$ position when the tip is closer to the samples. For scans of the samples containing a $2 \mu m$ diameter glass sphere (Figs. 1,3) $z_{\text {min }}$ is chosen so that the distance between the lower point of the oscillating tip and the glass substrate is keep constant for all $x$. The tip scanning movement is thus not perfectly parallel along the $x$ direction, but bends slightly. For the sample consisting of a 10 and a $30 \mathrm{~nm}$ sphere (Fig. 4), the scan trajectory follows $x$ exactly and $z_{\min }$ is always the same.

Occasionally, the near fields will be of interest, in particular the near field enhancement, i.e. the maximum of the electric field module in the proximity of the tip apex normalized to the module of the incident electric field. More relevant to experimental work, we also consider the scattered far fields. A rotationally symmetric lens of numerical aperture 0.342 whose axis is placed in the backscattering direction collects elastically scattered radiation. We model the lens as ideal, in the sense that it converts without power losses a spherical wave at the coordinate origin into a beam of constant phase at the output plane of the lens. We neglect any effects of further propagation or additional optical devices on the resulting beam. A planar photodetector perpendicular to the incoming radiation serves to convert the optical into an electrical signal. Precisely the different relationships between scattered fields and photodetector response distinguish heterodyne interferometric from noninterferometric detection schemes, as described by Eq. (3) and Eq. (2) respectively. For the interferometric case, the scattered signal $E_{s c a}$ is mixed with a reference planar wave of identical propagation direction but significantly stronger amplitude and a slightly shifted frequency. The polarization of the reference planar wave selects the p-polarized, in plane ( $x z$ plane) component of the scattered electric fields incident onto the detector. Notice that the considered scenarios are symmetric with respect to the $x z$ plane and an integral analogous to Eq. (3) cancels for the out-of-plane field component. As Eq. (3) depends on the strength of the reference beam, no attempt is made to compare the absolute strengths of the interferometric and non-interferometric signals. While the discussion in this paper mostly focuses on the absolute signal amplitude $\left|S_{n}\right|$, it is instructive to also consider the signed value $S_{n}$ for the non-interferometric case. In this case, each sign corresponds to one of the two possible phases, which are separated by $180^{\circ}$.

We first study the evolution of the signal as the tip-substrate distance is varied. These 
traces, also referred to as approach curves[28], are frequently helpful in understanding $a S N O M$ behavior. If the signal is dominated by the tip-substrate interaction in a small sample volume in the close proximity of the tip apex, as desired, the strength of the signal should significantly increase for distances smaller than the apex radius. Nonetheless, while approach curves are useful, a more complete $a S N O M$ analysis requires to implicitly include the imaging process[10]. We study the evolution of the signal for line scans of the patterned substrates. Notice that this requires a 2-dimensional movement of the the tip as it oscillates along $z$ and scans along $x$.

\section{RESULTS}

To illustrate the differences between heterodyne interferometric and non-interferometric schemes, we plot in Fig. 2 approach curves over the upper point of the $2 \mu m$ diameter homogeneous gold sphere, for different oscillations amplitudes and second and third harmonic demodulation. $\left|S_{n}\right|$ markedly increases for small tip substrate distance and both harmonics when the oscillation amplitude is small, a manifestation of the expected discrimination of the information from the tip-substrate interaction. Small oscillation amplitudes also result in a lower signal level, however, which often requires a compromise. For a given oscillation amplitude, the change of behavior for short distances is more clearly appreciated for the third than for the second harmonic, both for interferometric and for non-interferometric signals.

The former similarities notwithstanding, the heterodyne interferometric (Fig. 2(e-f)) and non-interferometric (Fig. 2(a-d)) approach curves are different. How changing the tip position affects the recorded signal clearly depends on the detection scheme used. The differences are perhaps more apparent at the second harmonic and large oscillation amplitudes. A more pronounced increase in $\left|S_{n}\right|$ is observed for short tip-substrate distances in the interferometric case. Notice also that the non-interferometric signal can equal zero, which suggests a change of sign as confirmed by plotting $S_{n}$ instead of $\left|S_{n}\right|$ (Fig. 2(c-d)).

We next consider the gold inclusion in the glass substrate. Fig. 1(b) shows the electric field modulus in the proximity of the apex for a given tip position[22]. The small gap between the tip and the inclusion distinctly exhibits the strongest fields. We include in Fig. 1(c) additional information on the behavior of the near and far fields. For this particular 
(a)

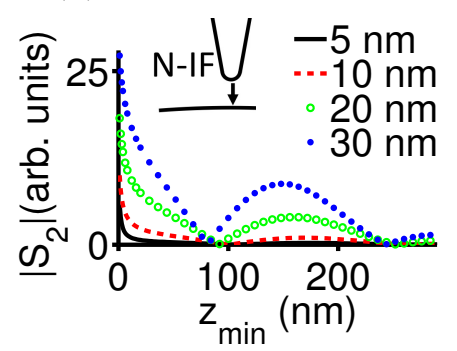

(b)



(c)

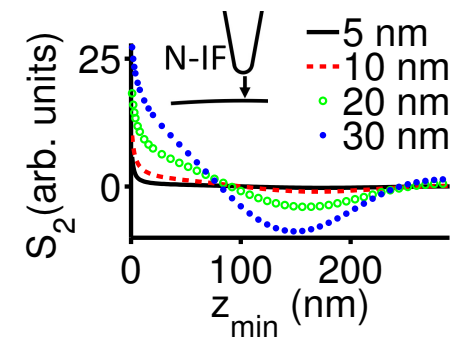

(d)

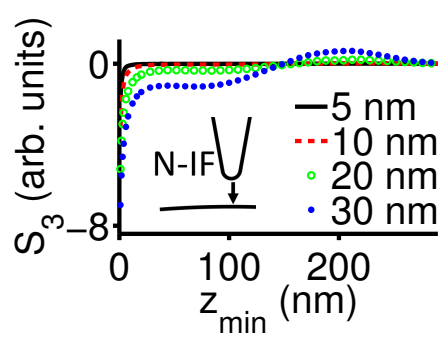

(e)

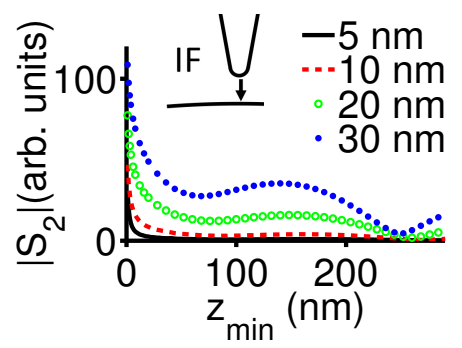

(f)

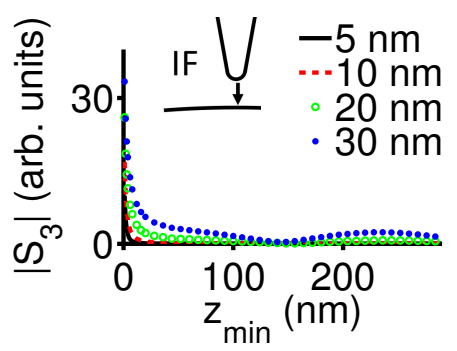

FIG. 2: Approach curves over the upper point of an spherical homogeneous gold substrate sphere $(2 \mu m$ diameter) for different oscillation amplitudes for either non-interferometric (a,b,c,d) or heterodyne interferometric schemes (e,f). $z_{\min }$ equals the tip apex-substrate distance at the lower oscillation point. (a,b,e,f) consider the modulus of the detected signal, while (c,d) include the sign information. (a,c,e) have been calculated for the second harmonic of the oscillation of the tip, and (b,d,f) for the third. Different arbitrary units are used for interferometric and non-interferometric values, as the latter depends on the reference beam intensity

geometry, but not in general [29], the near field enhancement broadly resembles the field distribution in the absence of the tip. Both are characterized by a clear maximum for $x$ close to 0 , corresponding to the position of the inclusion, with the most significant difference being the stronger fields in the presence of the tip. When the tip is at $x \sim 0$, the near field enhancement is $\sim 35.5$. In comparison, the scattered electric near field module at $1 \mathrm{~nm}$ above the substrate in the absence of the tip reaches a maximum of only about twice the excitation. The maximum field in the absence of the tip is not exactly at $x=0$ due to the 
oblique illumination.

The near field magnitude is relatively weak when the tip apex is situated above the bare substrate but increases strongly above a gold inclusion, particularly at $x=0$, which indicates an enhanced interaction [30-32]. In contrast, the non-interferometric, undemodulated far field signal $I_{d e t}$ is only weakly dependent on the tip position and shows no clear signature from the inclusion. Similarly, the modulus of the undemodulated heterodyne interferometric signal (not shown here) reveals a differently sloped but otherwise nearly featureless scan. However, a close correlation between the strength of this interaction and the detected far field signal would be convenient to interpret measurements in an active configuration.

Higher harmonic demodulation allows regaining high resolution information. We consider a fixed tip-sample distance of $1 \mathrm{~nm}$ at the lower oscillation point and an increasing oscillation amplitude. Fig. 3 shows second and third harmonic demodulated signal scans along $x$ according to Eq. (1) for both heterodyne interferometric and non-interferometric detection. Again, some common aspects emerge. A local extremum at $x \sim 0$ reveals the presence of the inclusion. It is especially distinct for small oscillation amplitudes and illustrates the $a S N O M$ capabilities for nanometer resolution[8] of subsurface objects[33, 34]. The traces for small oscillation amplitude presented here resemble the behavior of the interaction strength, as observed from the near field enhancement near the apex in Fig. 1(c).

Looking in closer detail, a narrow global maximum appears for all considered heterodyne interferometric detection traces (Fig. 3(e-f)) when the tip is situated directly above the gold inclusion. This maximum rides on a slowly varying background contribution whose average value and small slope depend on the oscillation amplitude. Thus, there is a consistent and relatively straightforward connection with the near field signal.

For non-interferometric detection, a clear global maximum of the $\left|S_{n}\right|$ signal - which should be indicative of the tip being close to the inclusion - appears only for certain experimental conditions (Fig. 3(a-b)). In other cases the extremum at $x \sim 0$ becomes even a local minimum. Notice also the often significant slope for large $x$. This slope could be related with the self-homodyne term $\left|E_{b k g}\right|\left|E_{o b j}\right| \cos \left(\Theta_{b k g}-\Theta_{o b j}\right)$ due to nearly constant background fields. The fields scattered by the isolated glass spherical substrate were substracted and thus do not contribute, but the large tip makes other significant contributions to be expected. Including strong scattering from a real substrate may lead to a more marked effect and more difficult to interpret non-interferometric images. 
(a)



(b)

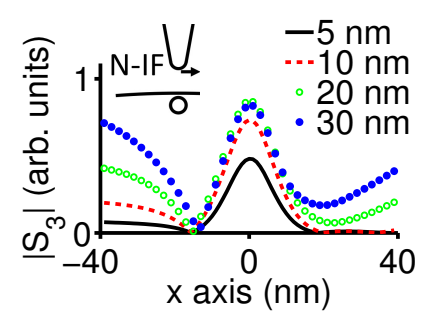

(c)

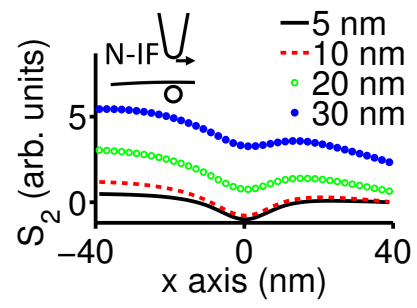

(d)

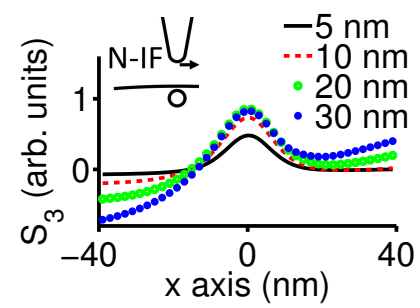

(e)

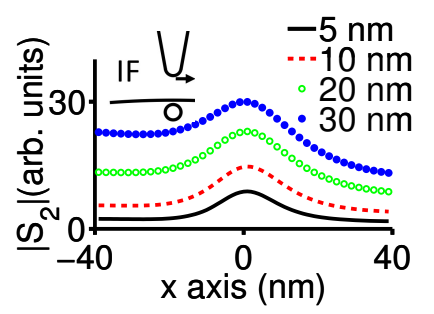

(f)

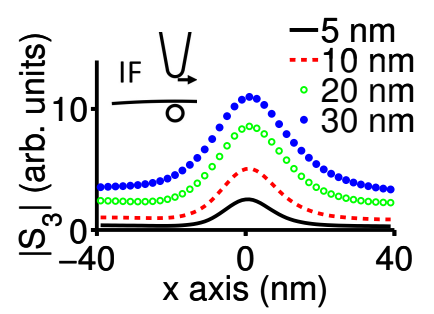

FIG. 3: Demodulated results for one dimensional scans of the gold spherical inclusion in the large glass sphere as in Fig. 1, considering different oscillation amplitudes, for either non-interferometric $(a, b, c, d)$ or heterodyne interferometric schemes $(e, f)$. (a,b,e,f) consider the modulus of the detected signal, while (c,d) include the sign information. (a,c,e) have been calculated for the second harmonic of the oscillation of the tip, and $(b, d, f)$ for the third. The arbitrary units are not comparable for interferometric and non-interferometric values, as the former depends on the reference beam. The distance to the substrate at the lower point of oscillation is kept at $1 \mathrm{~nm}$ during the complete scans. $x=0$ corresponds to the $x$ coordinate of the center of both the glass sphere and gold inclusion

Plotting the signed non-interferometric signal $S_{n}$ (Fig. 3(c-d)) results in a more regular evolution with oscillation amplitude. In this case, the scans for the second harmonic and all considered oscillation amplitudes show a local minimum for $x \sim 0$ and, outside the central region, a negative slope for increasing $x$. They become a maximum and a positive slope for the third harmonic traces. Notice that sign of the slope and the nature of the extremum depends on the reference angle $\Theta_{l i}$ of the lock-in amplifier - any 180 degree shift corresponding to a sign change

Last we consider scans over a sample consisting on two gold spheres of 10 and $30 \mathrm{~nm}$ radius. 
(a)

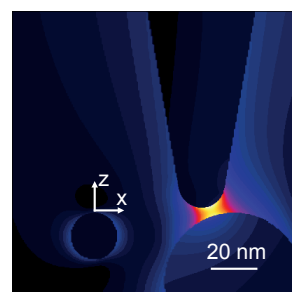

(b)

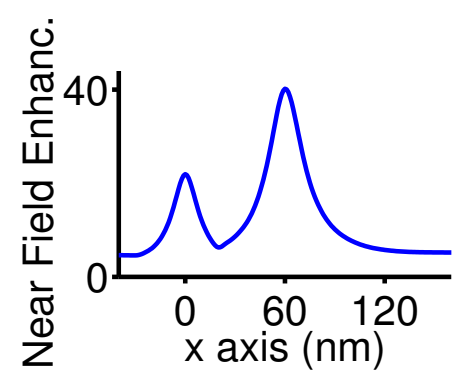

(c)

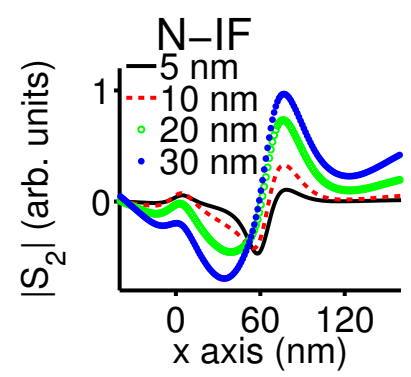

(d)

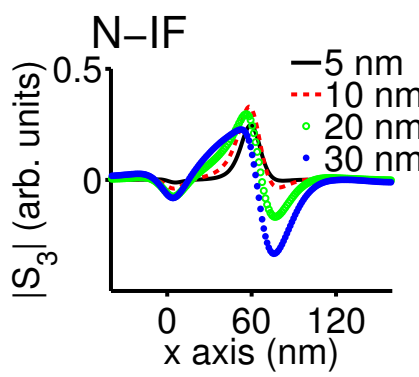

(e)

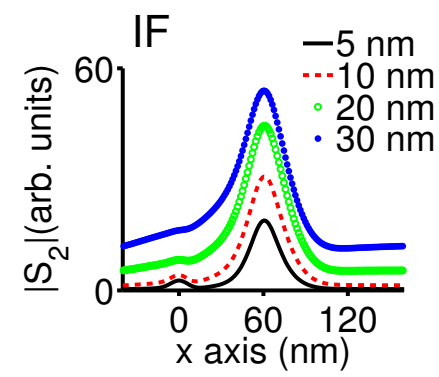

(f)

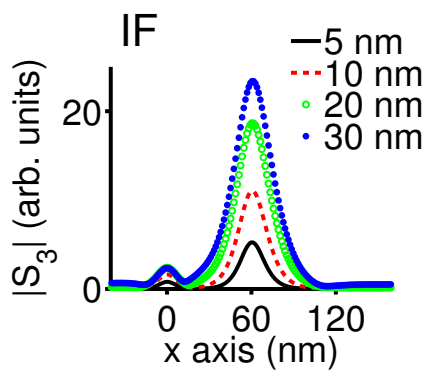

FIG. 4: (a) Example of the electrical field modules for the tip close to the substrate composed by a 10 and a $30 \mathrm{~nm}$ radius gold sphere in vacuum. The $z$ axis is again parallel to the tip axis and the tip scans along the perpendicular $x$ axis. The center of the large and small sphere is at $z=-31 \mathrm{~nm}, x=60 \mathrm{~nm}$ and $z=-11 \mathrm{~nm}, x=0 \mathrm{~nm}$, respectively. The strongest near fields, in white, are $\sim 19.5$ times larger than the excitation field. The color scheme is linear, with black corresponding to 0 field strength. (b) Near field enhancement for geometry in (a) when a nonoscillating tip scans the sample along $x$ for the lower point of the tip at a fixed $z 2 n m$ over the upper point of the spheres. In both (a) and (b) the contribution from the planar wave used as excitation have been substracted. (c-d) Signed non-interferometric $S_{n}$ and (e,f) heterodyne interferometric module $\left|S_{n}\right|$ signal for the same geommetry as the oscillating tip scans the sample along $x$. For all $x$, the lower point of the tip during each oscillation is at the same $z, 2 n m$ over the upper point of both spheres. (c-e) refers to the second and (d-f) to the third harmonic, in both cases for different oscillation amplitudes. The arbitrary units are different for the interferometric and non-interferometric cases. 
We have considered the spheres placed in vacuum, which is not realistic but simplifies the numerical burden and should nonetheless give important insights. Fig. 4(a) represents a snapshot of the electric fields module for a given tip position, while Fig. 4(b) shows the near field enhancement as the tip moves along $x$ without oscillating. The lower point of the tip apex is kept constant at $2 \mathrm{~nm}$ over the upper point of the spheres. The obtained trace presents marked maxima when the tip is close to any of the gold spheres, with a clearly stronger response in the case of the larger sphere. Stronger fields for larger radius is unsurprising from previous work[35], although a more detailed study for the present case would require to consider the spectral response.

Fig. 4(c-f) shows the demodulated far field signal as the oscillating tip scans the sample, with the lower point of the tip apex during each oscillation cycle at the same $z$, corresponding to $2 \mathrm{~nm}$ above the upper point of the spheres, for all $x$. For non-interferometric detection, the signal is difficult to interpret. A change of the signal appears when the tip approaches any of the two gold spheres, but the traces often differ markedly from the behavior of the near fields in Fig. 4(b). Even for oscillation amplitudes as low as $5 \mathrm{~nm}$ and third harmonic demodulation a significant difference remains: while it is possible to associate an extremum with each sphere, the signed signal $S_{n}$ (Fig. 4(c-d)) reveals that one is a maximum and the other a minimum. For increased oscillation amplitude the resemblance between the second or third harmonic signal and the near field enhancement can be very small.

In contrast, for the heterodyne interferometric signal (Fig. 4(e-f)) two different $\left|S_{n}\right|$ maxima, one for each inclusion, are clear for all third harmonic traces and also for the second harmonic and low amplitudes. For the second harmonic and $\sim 30 \mathrm{~nm}$ amplitude the signature from the $10 \mathrm{~nm}$ radius inclusion is very weak, and indeed it is significantly weaker than when the large spherical glass substrate was considered. Nonetheless, a slight inflexion in the signal behavior due to the presence of the smallest inclusion is still present. The different maxima sometimes ride on a non-negligible additional contribution that varies slowly and remains moderately strong for large $z$. In conclusion, the described near field enhancement and demodulated signal behave much more similarly in the heterodyne interferometric case. 


\section{DISCUSSION AND CONCLUSIONS}

This paper uses simulations to better understand the influence of the detection scheme in $a S N O M$, and in particular differences between non-interferometric and heterodyne (or equivalent) interferometric schemes. We have discussed in previous works $[5,22,36]$ the detrimental effects of simplifications such as using small tips. To increase the reliability of the obtained conclusions, we are interested in models that approach typical experimental conditions as closely as possible. An important step towards this objective is the inclusion of large tips and, in some of the treated cases, extended substrates: The 1400 $\mathrm{nm}$ tip used for the approach curves is several wavelengths long and numerically close to the even longer tips of typical experiments. The somewhat shorter tips $(500 \mathrm{~nm})$ considered in the onedimensional scans help to reduce the numerical complexity of the simulation, while being likely long enough to capture most significant phenomena. The homogeneous spheres of $2 \mu m$ diameter serve as an approximately flat substrate in the proximity of the apex.

Some differences remain between a large sphere and the more typical semi-infinite flat substrate, such as the existence of whispering gallery modes in the former. Real samples are significantly more complicated than either, and we chose the large sphere for numerical simplicity. In this respect, we discussed in the introduction how non-interferometric detection is affected by any constant (independent of tip position) contributions to the scattered fields, whereas heterodyne interferometric detection typically is not. This contribution can depend strongly on sample details, and may significantly complicate image interpretation in realistic experiments. To reduce the effect in the present study, we subtracted the constant backscattering component from the $2 \mu m$ diameter spheres in isolation.

For the non-interferometric scheme, we can simply consider just the amplitude $\left|S_{n}\right|$ at the dual-phase lock-in amplifier according to Eq. (1) or we can in addition associate $180^{\circ}$ phase jumps with a sign change. The latter implies working with negative values, conceptually not a problem because our demodulated signal corresponds to the coefficient of a Fourier series, which can be non-positive. The simulated heterodyne interferometric results focus on the amplitude of the magnitude measured by the lock-in amplifier. Still, for accurate results we must consider the phase of the scattered optical fields. Notice that it is necessary to include the effect of the collecting lens geometry to correctly predict the phase distribution on the photodetector. 
The simulated approach curves (Fig. 2) offer a direct connection to much work done in apertureless near-field optical microscopy. They serve to illustrate how the dependence of the signal amplitude $\left|S_{n}\right|$ on tip position is different for heterodyne interferometric and non-interferometric schemes.

The simulation of non spatially homogeneous samples allows to study the differences in more detail. We consider as experimentally favorable a monotonous dependence between the final signal and the strength of the tip apex-substrate interaction, which we have associated to the near field enhancement near the tip apex. Under this criterion, and remembering the results displayed in Fig. 1(c) and Fig. 4(b), a strong signal should be obtained when the tip is directly over a gold sphere and a weaker and nearly constant value when far away.

The obtained non-interferometric signal often departs from expectations. It frequently seems convenient to consider the sign and treat $S_{n}$ instead of $\left|S_{n}\right|$; still, even then the results are often difficult to interpret. This difficulty is already apparent in the strong slope of some of the traces for the inclusion in glass, but it is particularly evident for the sample consisting of two gold spheres.

The heterodyne interferometric results are significantly more encouraging, The correspondence between near field enhancement and far fields is not exact, with notably the maxima from the small inclusion in Fig. 4 more marked for the near field enhancement, but it seems nonetheless quite satisfactory and a straightforward interpretation of the demodulated results was possible. For other geometries more complex images will likely result — for example, if the background contribution to the demodulated signal becomes sufficiently large and adds destructively to the signal of interest. In this case, it may be convenient to retain the complex valued demodulated $S_{n}$ signal, and not only its amplitude as in the present discussion. This should facilitate spacial filtering of the recorded image. Preliminary results appear to support these assertions.

In summary, we have contrasted non-interferometric and heterodyne interferometric detection schemes for apertureless SNOM. Both are capable of extracting local information about the chosen substrate. The correspondence between the tip-substrate interaction strength and the modulus of the measured signal is more robust to changes on the scanning conditions and more directly interpretable for heterodyne interferometric detection. For non-interferometrically detected signals, considering the sign of the signal, as obtained from 
the phase of the lock-in output signal, seems beneficial.

[1] F. Zenhausern, Y. Martin, H. K. Wickramasinghe, Scanning interferometric apertureless microscopy: Optical imaging at 10 angstrom resolution, Science 269 (1995) 1083-1085.

[2] B. Knoll, F. Keilmann, Near-field probing of vibrational absorption for chemical microscopy, Nature 399 (1999) 134-137.

[3] R. Hillenbrand, F. Keilmann, Complex optical constants on a subwavelength scale, Phys. Rev. Lett. 85 (2000) 3029-3032.

[4] A. J. Huber, F. Keilmann, J. Wittborn, J. Aizpurua, R. Hillenbrand, Terahertz near-field nanoscopy of mobile carriers in single semiconductor nanodevices, Nano. Lett. 8 (2008) 37663770 .

[5] R. Esteban, R. Vogelgesang, K. Kern, Tip-substrate interaction in optical near field microscopy, Phys. Rev. B 75 (2007) 195410.

[6] J. N. Walford, J. A. Porto, R. Carminati, J.-J. Greffet, P. M. Adam, S. Hudlet, J.-L. Bijeon, A. Stashkevich, P. Royer, Influence of tip modulation on image formation in scanning nearfield optical microscopy, J. Appl. Phys. 89 (2001) 5159-5169.

[7] R. Esteban, R. Vogelgesang, J. Dorfmüller, A. Dmitriev, C. Rockstuhl, C. Etrich, K. Kern, Direct near-field optical imaging of higher order plasmonic resonances, Nano Letters 8 (2008) $3155-3159$.

[8] T. Taubner, R. Hillenbrand, F. Keilmann, Performance of visible and mid-infrared scatteringtype near-field optical microscopes, J. Microscopy 210 (2003) 311-314.

[9] L. V. Stebounova, Y. E. Romanyuk, R.-G. Dengel, S. R. Leone, Size-dependent near-field coupling observed from inn nanodots grown on nitrided sapphire, Chem. Phys. Lett. 443 (2007) 333-336.

[10] R. Fikri, T. Grosges, D. Barchiesi, Apertureless scanning near-field optical microscopy: Numerical modeling of the lock-in detection, Opt. Comm. 232 (2004) 15-23.

[11] I. Kopf, J.-S. Samson, G. Wollny, C. Grunwald, E. Bründermann, M. Havenith, Chemical imaging of microstructured self-assembled monolayers with nanometer resolution, J. Phys. Chem. C 111 (2007) 8166-8171.

[12] L. Gomez, R. Bachelot, A. Bouhelier, G. P. Wiederrecht, S.-H. Chang, S. K. Gray, F. Hua, 
S. Jeon, J. A. Rogers, M. E. Castro, S. Blaize, I. Stefanon, G. Lerondel, P. Royer, Apertureless scanning near-field optical microscopy: A comparison between homodyne and heterodyne approaches, J. Opt. Soc. Am. B 23 (2006) 823-833.

[13] S. Aubert, A. Bruyant, S. Blaize, R. Bachelot, G. Lerondel, S. Hudlet, P. Royer, Analysis of the interferometric effect of the background light in apertureless scanning near-field optical microscopy, J. Opt. Soc. Am. B 20 (2003) 2117-2124.

[14] R. Esteban, Apertureless SNOM : Realistic Modeling of the Imaging Process and Measurements of Resonant Plasmonic Nanostructures., Ph.D. thesis, EPFL, 2007.

[15] F. Formanek, Y. De Wilde, L. Aigouy, Analysis of the measured signals in apertureless nearfield optical microscopy, Ultramicroscopy 103 (2005) 133-139.

[16] B. Deutsch, R. Hillenbrand, L. Novotny, Near-field amplitude and phase recovery using phasesifting interferometry, Opt. Exp. 16 (2008) 494-501.

[17] M. Labardi, O. Tikhomirov, C. Ascoli, M. Allegrini, Balanced homodyning for apertureless near-field optical imaging, Rev. Sci. Instrum. 79 (2008) 033709.

[18] P. G. Gucciardi, G. Bachelier, M. Allegrini, Far-field background suppression in tip-modulated apertureless near-field optical microscopy, J. App. Phys. 99 (2006) 124309.

[19] C.-H. Chuang, Y.-L. Lo, An analysis of heterodyne signals in apertureless scanning near-field optical microscopy, Opt. Exp. 16 (2008) 17982-18003.

[20] D. Roy, S. Leong, M. E. Welland, Dielectric contrast imaging using apertureless scanning near-field optical microscopy in the reflection mode, Journal of the Korean Physical Society 47 (2005) 140-146.

[21] W. Chen, A. Kimil, A. Kirilyuk, T. Rasing, Apertureless snom study on gold nanoparticles: Experiments and simulations, Phys. Status. Solidi B 247 (2010) 2047-2050.

[22] R. Esteban, R. Vogelgesang, K. Kern, Full simulations of the apertureless scanning near field optical microscopy signal: achievable resolution and contrast, Opt. Express. 17 (2009) $2518-2529$.

[23] S. Goumri-Said, L. Salomon, J. P. Dufour, F. de Fornel, A. V. Zayats, Numerical simulations of photon scanning tunneling microscopy: role of a probe tip geometry in image formation, Opt. Commun. 244 (2005) 245-258.

[24] T. Sannomiya, J. Vörös, C. Hafner, Symmetry decomposed mmp calculation of plasmonic particles on substrate for biosensing applications, J. Comput. Theor. Nanosci. 6 (2009) 749- 
756.

[25] D. E. Aspnes, A. A. Studna, Dielectric functions and optical parameters of si, ge, GaP, GaAs, GaSb, InP, InAs, and InSb from 1.5 to 6.0 eV, Phys. Rev. B 27 (1983) 985-1009.

[26] P. B. Johnson, R. W. Christy, Optical constants of the noble metals, Phys. Rev. B 6 (1972) 4370-4379.

[27] T. Evers, H. Dahl, T. Wriedt, Extension of the program 3d MMP with a fifth order gaussian beam, Electr. Lett. 32 (1996) 1356-1357.

[28] B. Knoll, F. Keilmann, Enhanced dielectric contrast in scattering-type scanning near-field optical microscopy, Opt. Commun. 182 (2000) 321-328.

[29] A. García-Etxarri, I. Romero, F. J. García de Abajo, R. Hillenbrand, J. Aizpurua, Influence of the tip in near-field imaging of nanoparticle plasmonic modes: Weak and strong coupling regimes, Phys. Rev. B. 79 (2009) 125439.

[30] M. B. Raschke, C. Lienau, Apertureless near-field optical microscopy: Tip-sample coupling in elastic light scattering, Appl. Phys. Lett. 83 (2003) 5089-5091.

[31] Y. Abate, A. Schwartzberg, D. Strasser, S. R. Leone, Nanometer-scale size dependent imaging of cetyl trimethyl ammonium bromide (ctab) capped and uncapped gold nanoparticles by apertureless near-field optical microscopy, Chem. Phys. Lett. 474 (2009) 146-152.

[32] I. Notingher, A. Elfick, Effect of sample and substrate electric properties on the electric field enhancement at the apex of spm nanotips, J. Phys. Chem. B 109 (2005) 15699-15706.

[33] N. Anderson, P. Anger, A. Hartschuh, L. Novotny, Subsurface raman imaging with nanoscale resolution, Nano Lett. 6 (2006) 744-749.

[34] R. Carminati, J.-J. Greffet, Influence of dielectric constrast and topography on the near field scattered by an inhomogeneous surface, J. Opt. Soc. Am. A 12 (1995) 2716-2725.

[35] H. Xu, J. Aizpurua, M. Käll, P. Apell, Electromagnetic contributions to single-molecule sensitivity in surface-enhanced raman scattering, Phys. Rev. E 62 (2000) 4318-4324.

[36] R. Esteban, R. Vogelgesang, K. Kern, Simulation of optical near and far fields of dielectric apertureless scanning probes, Nanotechnology 17 (2006) 475-482. 